\title{
Why can’t you get pregnant? Causes of Infertility
}

\author{
Afa Bayramova* \\ Department of Gynaecology, Yaroslavl State Medical University, Russia
}

Received: April 18, 2018; Published: June 06, 2018

*Corresponding author: Afa Bayramova, Department of Gynaecology, Yaroslavl State Medical University, Yaroslavl Regional Perinatal Center, Yaroslavl, Russia, Tel: +79099555044; Email: yourdoctor.md@gmail.com

\begin{abstract}
Pregnancy is one of the most anticipated periods in the life of most women. Many of them, planning a child, try to make conception come, as soon as possible. But sometimes there are serious problems with fertility. Percent of women aged 15-44 with impaired fecundity in the USA is $12.1 \%$ [1]. Infertility in a married couple is usually diagnosed if pregnancy does not occur within a year of regular sexual activity without the use of contraception. To identify the causes of infertility, both partners should be examined, since problems can be in men, women, and even in both (combined infertility). If pregnancy has not occurred even once, then talk about primary infertility. Secondary infertility is diagnosed if a woman has already experienced a pregnancy earlier (whatever it ends). There is absolute infertility due to defects in the reproductive system (surgical removal of organs and glands or congenital mal development) and relative, the causes of which can be eliminated by the forces of modern medicine.
\end{abstract}

Keywords: Infertility; Gynecology; Endocrinology; Surgery

\section{Introduction}

A couple's examination of infertility begins with a conversation with both spouses, as the reproductive capacity in men is reduced in $30-40 \%$ of cases of infertile marriage. It starts with the fact that even with normal reproductive health of both partners; it is not always possible to conceive a child according to the schedule, which for themselves was composed by future parents. The process of fertilization depends on many different factors. You need to remember about certain points that significantly reduce the chance of conception [2].

They include:

1. Rare, irregular, or conversely, too frequent sex. In the first case, you can "not get" into ovulation. In the second, a man just does not have enough time to accumulate a sufficient number of mature sperm for fertilization.

2. Regular intake of alcohol and smoking. Bad habits reduce the chance of successful fertilization.

3. Taking certain medications (antibiotics, antidepressants, pain medications). May reduce the quality of sperm in men; disrupt the process of maturation of the egg in women.

4. Irrational eating. The lack of vitamins, minerals and amino acids can lead to a decline in fertility.

5. All of the above factors can significantly affect why you cannot get pregnant. Eliminating them, the chances of conception increase.
6. Causes of female infertility.

7. Modern science studies the causes leading to female infertility, and distinguishes the following groups [3].

8. Pipe and tube-peritoneal factor: the obstruction of fallopian (uterine) tubes physically blocks the mature egg to meet the spermatozoon.

9. Gynecological diseases. Endometriosis, chronic or acute inflammatory processes in the pelvic organs often make successful conception impossible. They break the patency of the fallopian tubes or make it impossible to implant the embryo into the wall of the uterus.

10. Premature exhaustion of the ovarian reserve (early menopause) is another reason for not being able to conceive. Fertilized eggs do not ripen in each cycle.

11. Disorders in the endocrine system: hormonal imbalance can adversely affect the maturation and functionality of the egg. Deviations in the development of a hormone in different ways affect the ability of conception. Hormonal causes of infertility can be caused by violations of the normal blood levels of such major hormones as FSH (follicle-stimulating hormone), LH (luteinizing hormone), prolactin, estradiol, progesterone, testosterone, DEA-sulfate and others. For example, the excess in the body of prolactin does not allow the fertilized egg to attach to the wall of the uterus [4]. 
12. Congenital or acquired pathology of the uterus: underdevelopment, duplication, internal septum, shape features, postoperative scars, uterine tumor (myoma), or endometrial pathology.

13. Immunological incompatibility: the development of an antibody to partner spermatozoa by the female body, to the tissues of its own ovary, endometrium, excessive affinity of the partners for tissue compatibility antigens (HLAcompatibility), cytokine imbalance, negative postcoital test.

14. We cannot discount the age factor: on average, the peak of female fertility falls on 20-25 years; it remains high enough to 35 years, and after a fairly fast extinction down to menopause. The female organism does not produce new sex cells, but only induces maturation of the available from birth in the "ovarian reserve". With age, irreversible changes accumulate in it. Statistically, a woman of thirty left $12 \%$ of an ovarian reserve, in a forty-year-old woman only $3 \%$. Nevertheless, it is impossible to strictly limit the age limits of fertility in each individual case. Conception in adulthood is not uncommon for a woman.

15. Diseases of the thyroid gland, adrenal glands, pituitary gland cause infertility as a side effect of hormonal imbalance. Diabetes mellitus, kidney and liver disorders, systemic diseases can become an obstacle to pregnancy, and in cases of severe leakage, doctors can advise you to abandon the idea of pregnancy in the name of preserving the woman's health.

16. Infectious, asymptomatic sexual infections of a chronic nature are the cause of internal inflammatory processes, which may result in the adhesion of the fallopian tubes or endometritis [5].

17. Excessive or insufficient body weight also leads to violations of the hormonal balance. Adipose tissue is the organ that produces estrogen. Lack of adipose tissue creates a deficiency of estrogen and leads to disruption of the menstrual cycle, in extreme cases, even to the cessation of menstruation. Excess weight and the accompanying excess of estrogen can also interfere with the onset of pregnancy.

18. Unbalanced diet, lack of vitamins and microelements necessary for the reproductive system, can also be the cause of non-pregnancy. For example, without folic acid, the proper development of the fetal nervous system at the earliest stage is impossible.

19. Women who smoke tobacco are at risk. Nicotine and tar contained in tobacco smoke, inhibit the production of estrogen, worsen blood circulation in the uterus and its shell, and reduce the susceptibility of the endometrium, slowing the transport of embryos.
20. Separately, infertility, developing against a background of grosses congenital anomalies of the genital organs, which may be a consequence of genetic pathology and chromosomal diseases. Such forms of infertility do not lend themselves to treatment. Pregnancy can only be achieved with the help of assisted reproductive technologies.

The so-called "false infertility" occurs when you consciously or unconsciously fail to comply with all the conditions necessary for the onset of pregnancy [6]. Including:

1. Simultaneous protection from pregnancy and infertility treatment,

2. Douching after sexual intercourse for hygienic purposes,

3. Sexual intercourse only in the second half of the cycle, after raising the basal temperature.

\section{What to do?}

Modern medicine successfully copes with many types of infertility. In each case, the methods of diagnosis and therapy will differ [7]. After identifying the cause, they try to eliminate or neutralize it. Many believe that infertility treatment is necessarily associated with surgery, but this is not true. So, for sexual infections antimicrobial agents and antibiotics are prescribed, and hormonal preparations are used to correct the hormonal balance.

\section{Prevention}

There are factors that cannot be prevented. For example, it may be congenital abnormalities of the uterus. However, some situations leading to female infertility can be avoided [8].

a. Use barrier contraception to reduce the risk of sexually transmitted diseases;

b. Visit the gynecologist at least once a year;

c. Visit the doctor for signs of inflammation of the ovaries, urinary tracts, painful sensations in the lower abdomen, cycle disorders-timely access to a specialist will allow to detect and treat inflammation of the pelvic organs in time and thus avoid adhesions and obstruction of the fallopian tubes.

d. Take antioxidants to protect eggs from negative environmental effects

e. Adjust your weight within reasonable limits to avoid chronic diseases (hypertension, diabetes, etc.) that complicate the onset of pregnancy.

The existing variety of factors responsible for the violation of the reproductive function requires compliance with standardized methods for examining patients, selection criteria and choice of 
therapy, which significantly increases the effectiveness of the treatment [9]. A couple's examination of infertility begins with a conversation with both spouses, as the reproductive capacity in men is reduced in $30-40 \%$ of cases of infertile marriage [10].

Terms of examination for infertility should not exceed 2-3 months, and treatment 2 years from the time of contact with the clinic. A married couple may be warned that the average pregnancy rate after infertility treatment does not exceed $40 \%$ and ranges from 20 to $80 \%$, depending on the nature of the reproductive harm. It has now been established that after a complete clinical and laboratory examination in $5-10 \%$ of couples, the cause of infertility remains unclear. At the first conversation to spouses it is necessary to explain that without a full phased examination; only after 1-2 visits to the doctor it is not necessary to count on the desired result [11].

The algorithm of examination in female infertility primarily involves the analysis of clinical and anamnestic indicators. When interviewing, attention should be paid to the duration of infertility and the connection of the violation of reproductive function with any factors. Describe in detail the nature of menstrual function, the change in body weight in a short period of time, the presence of secretions from the mammary glands. In addition, the psychological situation in the family is taken into account.

Much attention should be paid to previous diseases, surgical interventions, since the presence in the past of any gynecological operations-ovarian cysts, ectopic pregnancy, surgical treatment of the cervix, abortion, complicated delivery-can lead to infertility [12]. Find out whether the woman was diagnosed with sexually transmitted diseases (chlamydia, urea plasmosis, gonorrhea, viral infection, etc.), as their role in the development of the acquired pathology of the fallopian tubes is known.

In the WHO studies, it was noted that in almost half of the women examined for infertility, the inflammatory processes of the genitals were of gonococcal etiology, the rest of them revealed chlamydial, mycoplasmal, viral and other unspecific infections. The prevalence of Chlamydia infection has increased dramatically in recent years. This is due in part to the widespread introduction of more accurate methods of identifying the pathogen, but also reflects a high level of infection.

Deserves attention to the previous survey of women about infertility, methods of previous treatment, drugs used earlier, due to the fact that some drugs can cause temporary or permanent violation of the processes of ovulation [13]. Evaluation of the childbearing function includes elucidating the total number and outcomes of previous pregnancies in women with secondary infertility, particular importance attached to their complications, as this category of women has increased risk of pathology of the uterus and fallopian tubes of inflammatory genesis.
There are methods of regulating fertility, which have ever been applied by a woman. Some epidemiological studies have reported an increased risk of developing inflammation of the genitals with intrauterine contraception. However, it should be borne in mind that for the development of tubal infertility the most important factors are the number of sexual partners and the incidence of sexually transmitted diseases.

Gynecological examination provides a large amount of information and contributes to the correct verification of the diagnosis. Colposcopy or microcolposcopy is mandatory methods of examination at the first examination of the patient, they reveal signs of colpitis, cervicitis, endocervicitis and erosion of the cervix, which can cause infertility and can be a sign of chronic genital infection [14].

Functional diagnostic tests are used to assess ovarian hormonal activity and the presence of ovulation. The basal temperature chart is one of the most accessible methods of indicating the occurrence of ovulation. Sign of the ovulatory cycle are: two-phase nature of the temperature, with "zapping" on the day of ovulation by $0.2-0.3{ }^{\circ} \mathrm{C}$ and a rise in temperature in the luteal phase of the cycle compared with folliculin at 0.5 $0.6^{\circ} \mathrm{C}$ for duration of phase II of at least $12-14$ days. Shortening of the II phase of the cycle, a slow, small rise in temperature, a "stepped" type of curve indicate a deficiency in the function of the yellow body.

In the absence of ovulation, the basal temperature is monophasic. However, the presence of a two-phase nature of rectal temperature is not an indisputable proof of the ovulation that has occurred, just as monophasic nature does not prove that ovulation is absent. The confirmation of ovulation is also the level of progesterone, determined on the $20^{\text {th }}-24^{\text {th }}$ day of the cycle. For the diagnosis of ovulation, ultrasound is used with repeated scanning of the ovary to observe the growth of the follicle and its rupture, and an endometrial biopsy from its secretory changes [15].

Hormonal examination is carried out according to the indications. In the case of determining elevated indicators, it is necessary to re-determine the level of hormones. Evaluation of the function of the adrenal cortex is carried out according to the level of excretion of dehydroepiandrosterone sulfate. With a regular rhythm of menstruation, it is justified to determine the level of prolactin, testosterone, cortisol and the level of thyroid hormones in the blood plasma to the follicular phase on the $5^{\text {th }}$ $7^{\text {th }}$ day of the menstrual cycle. In the II phase, on the $20-22^{\text {nd }}$ day, the definition of progesterone is recommended in order to assess the usefulness of ovulation and the function of the yellow body.

With oligomenorrhea and amenorrhea, it is mandatory to determine the level of all hormones: FSH, LH, prolactin, estradiol, 
testosterone, DEA-C, TTG, T3, and T4. The experience gained in the clinic of infertility, indicates that a single determination of the basal level of hormones in the blood is not always informative. To clarify the state of the various parts of the reproductive system or to determine their reserve capacity allows hormonal tests. These include a progesterone test, a test with estrogens, clostilbegite, gonadoliberin, dexamethasone, and others [16].

In patients with inflammatory diseases of the genitals, it is necessary to take into account that various pathogens (chlamydia, mycoplasmas, viruses) can cause similar in clinical manifestations processes in the reproductive system: serouspurulent discharge from the cervical canal, hyperemia around the external throat (endocervicitis), erosion, pseudo-erosion of the cervix and urethritis. Viral infections of the reproductive system are among the most common and present diagnostic difficulties, since the infection can be latent for a long time. With clinical manifestation, there is a characteristic sign the presence of vesicles on the background of edematous and hyperemic mucosa, pronounced itching. Diagnosis is based on the detection of the antibody titer of herpes simplex virus in the blood serum or a separable cervical canal, vagina, aspirate from the uterine cavity [17]. Ultrasound is one of the most common methods of examining the reproductive system without invasive intervention. Detailed information about the status of the uterus and fallopian tubes can be obtained by using endoscopic diagnostic methods: laparoscopy and hysteroscopy. These methods are very valuable in the clinic of female infertility, as they allow not only to clarify the pathology of the uterus and tubes, but also to perform a surgical correction [18].

After the completion of the survey, the violations found differentiate and make a diagnosis, respectively, which is prescribed treatment. The issue of infertility therapy in a marriage is resolved after a comprehensive examination of both spouses. Since each spouse can be identified several causes of reproductive harm, treatment should begin with the reasons that are of paramount importance [19]. For patients with infertility, the optimal method of surgical treatment is operative laparoscopy if the cause of infertility is the adhesive process. With operative endoscopy, the adhesions are dissected, and the normal patency of the tubes is restored. In the absence of the effect of conservative and surgical treatment after 1 year, the application of the IVF method is shown [20].

The treatment of endometriosis is based on the concept of a combined approach: surgical and medication. Laparoscopy is necessary for this contingent of patients, as it allows diagnosing, treating endometriosis and assessing the condition of the tubes and ovaries. During the operation endocoagulation of endometrioid heterotopia is carried out, as well as removal of endometrioid cysts of the ovaries, which, as is known, are not amenable to conservative treatment. In the future, hormone therapy is used [21].
When detecting myomatous nodes, a conservative myomectomy is performed, as many studies have shown that removing even small-sized knots in women with infertility promotes the onset of pregnancy in $40 \%$. An important point is the choice of surgical access. Endoscopic intervention is preferable, since with this access there is practically no risk of developing an adhesion process in the small pelvis as an additional factor of reproductive harm.

When detecting retinal formations of the ovaries, they are enucleated with subsequent histological examination. If a true tumor of the ovary is suspected, ovarian resection is performed within a healthy tissue with an intra-operative pathomorphological examination to resolve the issue of the adequacy of the scope of the surgical intervention. Treatment of endocrine forms of infertility should be differentiated depending on the cause and duration of infertility, as well as concomitant diseases. In conclusion, it should be noted that the use of an integrated step-by-step approach in the investigation of numerous reproductive disorders contributes to the correct diagnosis of various factors of infertility, which in turn determines the effectiveness of assisting infertile couples.

\section{References}

1. Chandra A, Stephen EH (2010) Impaired fecundity in the United States 1982-1995. Fam Plann Perspect 30(1): 34-42.

2. Link PW, Darling CA (2006) Couples undergoing treatment for infertility: dimensions of life satisfaction. J Sex Marital Ther 12(1): 4659.

3. Hillis SD, Nakashima A, Marchbanks PA, Addiss DG, Davis JP (2014) Risk factors for recurrent Chlamydia trachomatis infections in women. Am J Obstet Gynecol 170(3): 801-806.

4. Marrazzo JM, Celum CL, Hillis SD, Fine D, De Lisle S, et al. (2007) Performance and cost-effectiveness of selective screening criteria for Chlamydia trachomatis infection in women. Implications for a national Chlamydia control strategy. Sex Transm Dis 24(3): 131-141.

5. Buck GM, Sever LE, Batt RE, Mendola P (2007) Life-style factors and female infertility. Epidemiology 8(4): 435-441.

6. Schwartz D, Mayaux MJ (2010) Female fecundity as a function of age: results of artificial insemination in 2193 nulliparous women with azoospermic husbands. Federation CECOS. N Engl J Med 306(7): 404406.

7. Eskenazi B, Gold EB, Samuels SJ, Wight S, Lasley BL, et al. (1995) Prospective assessment of fecundability of female semiconductor workers. Am J Ind Med 28(6): 817-831.

8. Swan SH, Elkin EP, Fenster L (2007) Have sperm densities declined? A reanalysis of global trend data. Environ Health Perspect 105(11): 1228-1232.

9. Davis RO, Rothmann SA, Overstreet JW (1992) Accuracy and precision of computer-aided sperm analysis in multicenter studies. Fertil Steril 57(3): 648-653.

10. Dunphy BC, Neal LM, Cooke ID (1989) The clinical value of conventional semen analysis. Fertil Steril 51(2): 324-329.

11. Wilcox LS, Mosher WD (1993) Use of infertility services in the United States. Obstet Gynecol 82(1): 122-127. 
12. Collins JA, Wrixon W, Janes LB, Wilson EH (2013) Treatmentindependent pregnancy among infertile couples. N Engl J Med 309(20): 1201-1206.

13. Tucker KE (2006) Reproductive toxicity of ovulation induction. Semin Reprod Endocrinol 14(4): 345-353.

14. Whittemore AS, Harris R, Itnyre J, Halpern J (2012) Characteristics relating to ovarian cancer risk: collaborative analysis of 12 US casecontrol studies. I. Methods. Collaborative Ovarian Cancer Group. Am J Epidemiol 136(10): 1175-1183.

15. Bristow RE, Karlan BY (2006) Ovulation induction, infertility, and ovarian cancer risk. Fertil Steril 66(4): 499-507.

16. Shapiro S (2005) Risk of ovarian cancer after treatment for infertility. N Engl J Med 332(19): 1301-1302.

17. Nielsen HC, Harvey-Wilkes K, MacKinnon B, Hung S (2007) Neonatal outcome of very premature infants from multiple and singleton gestations. Am J Obstet Gynecol 177(3): 653-659.
18. Kiely JL, Kleinman JC, Kiely M (2002) Triplets and higher-order multiple births. Time trends and infant mortality. Am J Dis Child 146(7): 862-868.

19. Wilcox LS, Kiely JL, Melvin CL, Martin MC (2006) Assisted reproductive technologies: estimates of their contribution to multiple births and newborn hospital days in the United States. Fertil Steril 65(2): 361366.

20. Callahan TL, Hall JE, Ettner SL, Christiansen CL, Greene MF, et al. (2014) The economic impact of multiple-gestation pregnancies and the contribution of assisted-reproduction techniques to their incidence. N Engl J Med 331(4): 244-249.

21. Hidlebaugh DA, Thompson IE, Berger MJ (2007) Cost of assisted reproductive technologies for a health maintenance organization. J Reprod Med 42(9): 570-574.

Your next submission with Juniper Publishers will reach you the below assets

- Quality Editorial service

- Swift Peer Review

- Reprints availability

- E-prints Service

- Manuscript Podcast for convenient understanding

- Global attainment for your research

- Manuscript accessibility in different formats

( Pdf, E-pub, Full Text, Audio)

- Unceasing customer service

Track the below URL for one-step submission https://juniperpublishers.com/online-submission.php 